\title{
THE
}

\section{Genotype and Phenotype in 12 additional individuals with SATB2-Associated Syndrome}

\author{
Yuri A. Zarate \\ Kalsner \\ Alice Basinger \\ Julie R. Jones \\ Chumei Li
}

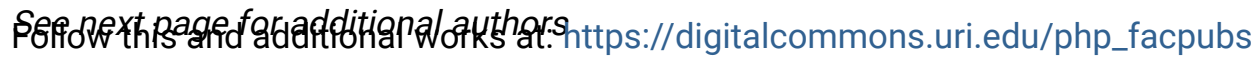

\section{The University of Rhode Island Faculty have made this article openly available.}

Please let us know how Open Access to this research benefits you.

This is a pre-publication author manuscript of the final, published article.

Terms of Use

This article is made available under the terms and conditions applicable towards Open Access Policy Articles, as set forth in our Terms of Use.

\section{Citation/Publisher Attribution}

Zarate, Y. A., Kalsner, L., Basinger, A., Jones, J. R., Li, C., Szybowska, M.,...Everman, D. B. (2017). Genotype and Phenotype in 12 additional individuals with SATB2-Associated Syndrome. Clinical Genetics, 92, 423-429. doi: $10.1111 / \mathrm{cge} .12982$

Available at: http://dx.doi.org/10.1111/cge.12982 


\section{Authors}

Yuri A. Zarate, Kalsner, Alice Basinger, Julie R. Jones, Chumei Li, Marta Szybowska, Zhou Luan Xu, Samantha Vergano, Aisling R. Caffrey, Cruz Velasco Gonzalez, Holly Dubbs, Elaine Zackai, Francisca Millan, Aida Telegrafi, Berivan Baskin, Richard Person, Jennifer L. Fish, and David B. Everman 


\section{Genotype and Phenotype in 12 additional individuals with $S A T B 2$-Associated Syndrome}

Yuri A. Zarate ${ }^{1}$, Louisa Kalsner ${ }^{2}$, Alice Basinger ${ }^{3}$, Julie R. Jones ${ }^{4}$, Chumei Li $^{5}$, Marta

Szybowska $^{5}$, Zhuo Luan $\mathrm{Xu}^{6}$, Samantha Vergano ${ }^{7}$, Aisling R. Caffrey ${ }^{8}$, Cruz Velasco

Gonzalez $^{9}$, Holly Dubbs ${ }^{10}$, Elaine Zackai ${ }^{10}$, Francisca Millan ${ }^{11}$, Aida Telegrafi ${ }^{11}$, Berivan

Baskin $^{11}$, Richard Person ${ }^{11}$, Jennifer L. Fish ${ }^{12}$, David B. Everman ${ }^{4}$

${ }^{1}$ Section of Genetics and Metabolism, University of Arkansas for Medical Sciences, Little Rock,

Arkansas

${ }^{2}$ Connecticut Children's Medical Center, Hartford, Connecticut

${ }^{3}$ Cook Children's Physician Network, Fort Worth, Texas

${ }^{4}$ Greenwood Genetic Center, Greenwood, SC

${ }^{5}$ Clinical genetics program, McMaster University Medical Center, Hamilton, Ontario, Canada

${ }^{6}$ Faculty of Health Sciences, McMaster University, Hamilton, Ontario, Canada

${ }^{7}$ Division of Medical Genetics and Metabolism, Children's Hospital of The King's Daughters,

Norfolk, Virginia

${ }^{8}$ Health Outcomes, Collage of pharmacy, University of Rhode Island, Kingston, Rhode Island

${ }^{9}$ Biostatistics Program, Department of Pediatrics, University of Arkansas for Medical Sciences,

Little Rock, Arkansas

${ }^{10}$ Children's Hospital of Philadelphia, Philadelphia, Pennsylvania

${ }^{11}$ GeneDx, Gaithersburg, Maryland 
${ }^{12}$ Department of Biological Sciences, University of Massachusetts Lowell, Lowell, Massachusetts

\section{Correspondence To:}

Yuri A. Zarate, M.D.

Arkansas Children's Hospital

1 Children's Way; Slot 512-22

Little Rock, AR 72202

Telephone: 501-364-2971, yazarate@uams.edu

Fax: 501-364-1564

Conflicts of Interest: F.M., A.T., B.B., and R. P. are employees of GeneDx, a wholly owned subsidiary of Opko Health, Inc. Y.A. Z. has worked as a clinical abstractor for GeneDx. D.B.E. and J.R.J are employees of the Greenwood Genetic Center, an institution that receives revenue from diagnostic testing performed in their Molecular Diagnostic Laboratory. L.K., A.B., C.L., M.S., Z.L.X., S.V., A.R.C., C.V. G., H.D., E.Z., and J.L.F. have no conflicts of interest to declare.

Acknowledgements: We thank Julie Nick and Sunitha Kenchey for their ongoing support with data management and all families for their generous collaboration. 


\begin{abstract}
SATB2-associated syndrome (SAS) is a multisystemic disorder caused by alterations of the SATB2 gene. We describe the phenotype and genotype of twelve individuals with 10 unique (de novo in 11 of 11 tested) pathogenic variants ( 1 splice site, 5 frameshift, 3 nonsense, 2 missense) in $S A T B 2$ and review all cases reported in the published literature caused by point alterations thus far. In the cohort here described, developmental delay with severe speech compromise, facial dysmorphism, and dental anomalies were present in all cases. We also present the third case of tibial bowing in an individual who, just as in the previous two individuals in the literature, also had a truncating pathogenic variant of $S A T B 2$. We explore early genotypephenotype correlations and reaffirm the main clinical features of this recognizable syndrome: universal developmental delay with severe speech impediment, mild facial dysmorphism, and high frequency of craniofacial anomalies, behavioral issues, and brain neuroradiographic changes. As the recently proposed surveillance guidelines for individuals with SAS are adopted by providers, further delineation of the frequency and impact of other phenotypic traits will become available. Similarly, as new cases of SAS are identified, further exploration of genotypephenotype correlations will be possible.
\end{abstract}

Keywords: $S A T B 2$, cleft palate, whole exome sequencing, tibial bowing 


\section{Introduction}

SATB2-associated syndrome (SAS; OMIM 612313) is a clinically recognizable syndrome characterized by neurodevelopmental and behavioral abnormalities, palatal and dental anomalies, dysmorphic features, and frequent skeletal pathology (1-3). From the neurodevelopmental perspective, developmental delay (DD)/intellectual disability (ID) with absent or limited speech development is virtually universal (1).A total of 42 individuals with SAS have been described thus far (1). Alterations in the SATB2 gene can result from a variety of different mechanisms that include contiguous deletions, intragenic deletions and duplications, translocations with secondary gene disruption, and point pathogenic variants (1). We, and others, have reported a total of 11 different SATB2 point pathogenic variants in 12 individuals with SAS (2-8). Here we report on our joint experience with a series of 12 previously unpublished individuals and their genotypes, which include 10 novel pathogenic variants.

\section{Materials and methods}

$\underline{\text { Individuals }}$

Individuals with probable pathogenic $S A T B 2$ variants were enrolled on the basis of their genotype. One individual (individual 23) was enrolled through the GeneDx laboratory after the referring provider was informed of research interest by Dr Zarate. The referring providers of individuals 1, 4, 10, 18, and 24 contacted Dr Zarate. For the rest of the individuals $(5,6,7,14$, 16, and 28), the family contacted Dr Zarate directly because of a new diagnosis of SAS made by genetic testing. All families reported herein agreed to share clinical information and were enrolled under a research clinical registry protocol approved by Institutional Review Board of 
the University Of Arkansas for Medical Sciences. For all individuals, medical records including laboratory results were reviewed as provided by the treating physician or the individual's caregiver directly. Supplementary information was also obtained from parents directly through a REDCap $^{\mathrm{TM}}$ questionnaire. All families who shared photographs signed consent for publication.

\section{$\underline{\text { Molecular studies }}$}

For 11 individuals in this cohort, whole exome sequencing (WES) was conducted on genomic DNA extracted from whole blood from the affected children and their parents. Exome sequencing was performed on exon targets isolated by capture using the Agilent Clinical Research Exome kit (Agilent Technologies, Santa Clara, CA). The sequencing methodology and variant interpretation protocol has been previously described (2). Pathogenic variants passing previously described filtering and prioritization criteria were confirmed using di-deoxy DNA sequence analysis using an ABI3730 (Life Technologies, Carlsbad, CA).A next generation sequencing (NGS) Intellectual Disabilities (ID) panel that includes 393 genes was performed on a single individual. All variants were confirmed by Sanger sequencing using standard protocols. In silico prediction tools for interpretation of the variants reported in this study included SIFT (http://sift.jcvi.org/), MutationTaster (http://www.mutationtaster.org/), Polyphen-2 (http://genetics.bwh.harvard.edu/pph2/), and Provean (http://provean.jcvi.org/index.php).

\section{Results}

\section{Phenotype}


We gathered data on 12 individuals diagnosed with SAS from 6 different countries (Table 1). These new SAS cases included 8 males (67\%), with a median current age of 6.5 years (range 2.514.5). None of the described individuals had a suspected clinical diagnosis. Their overall phenotypic features are described below.

Growth and neurodevelopment. Birth weight (mean $3.6 \mathrm{~kg}$ ) and length (mean $50.8 \mathrm{~cm}$ ) were reported to be normal in all 12 individuals. While postnatal growth retardation was described in only 4 individuals (33\%), feeding difficulties (gastroesophageal reflux, dysphagia, and need for special feeding nipples) during infancy were frequently documented $(9 / 12=75 \%)$ but none of the individuals required a gastrostomy feeding tube. Developmental delay was reported in all cases. Gross motor milestones were delayed as evidenced by the ages to reach early milestones such as rolling over (mean 5.2 months, range 3-10), sitting up (mean 8.2 months, range 6-14), and walking (mean 20.9 months, range 11-35). Speech was drastically affected as evidenced by the late age at first word (mean 19.8 months, range 13-42), the inability to speak in full sentences by any individual, and the high frequency of absent speech $(7 / 12=58 \%)$. An overfriendly or jovial personality was reported in 11 individuals (92\%). Behavioral abnormalities were common $(9 / 12=75 \%)$ with a high frequency of attention deficit/hyperactivity $(5 / 12=42 \%)$ and sleeping difficulties $(4 / 12=33 \%)$, among others (Supplementary table). Brain MRIs were performed in 9 individuals, 6 (67\%) of them with abnormal results: 3 had delayed myelination for age and 3 more had non-progressive white matter abnormalities. Five individuals had electroencephalograms (EEGs) performed to evaluate for possible seizures. Only a single individual was diagnosed with clinical seizures (absence) that were successfully treated with valproic acid. Another individual had EEG abnormalities (benign epilepsy with central temporal 
spikes in sleep) but did not require antiepileptic treatment. Lastly, abnormal gait and balance issues were reported in 3 individuals (25\%).

Craniofacial and dental. Dental anomalies were present in all individuals while palatal anomalies and micrognathia were often recognized (Table 1, Supplementary table). Mild facial dysmorphism was also documented in all individuals. While a specific dysmorphology pattern was not recognized, common features included a long and/or flat philtrum $(8 / 12=67 \%)$, deeply set eyes $(6 / 12=50 \%)$, and a thin vermillion of the upper lip (4/12=33\%) (Fig. 1).

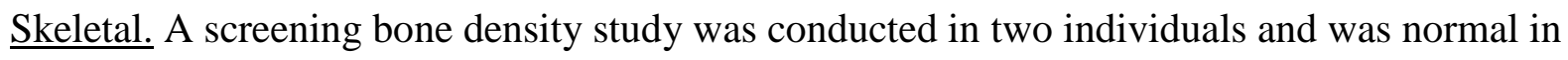
both cases (SATB2-10 at age 13 years, total body Z score of -1.2 SD, and SATB2-24 at age 6 years, total body Z-score of -1.1SD). An skeletal deformity was documented in a single individual. Individual SATB2-07 was diagnosed with bilateral anterior tibial bowing at age 7 years (16 degrees) but has not required further medical or surgical management (Supplemental Fig. 1).

Other medical issues. Sialorrhea was present in 5 individuals (42\%) with at least one individual needing Botulinum injections into the salivary glands. Individuals SATB2-10 and SATB2-14 were both noted to have broad thumbs. The latter also with $5^{\text {th }}$ finger clinodactyly, and lower limb asymmetry. The rest of the medical history was otherwise reported as normal in all individuals.

\section{Genotype}

In all 11 WES-trio cases, the variants were reported to be de novo. The 10 novel variants (2 individuals found to have the p.R429Q variant, one individual with the previously reported p.R283* variant) have not been reported in the Human Gene Mutation Database (HGMD) or any public database (dbSNP, ClinVar, Exome Variant Server, and Exome Aggregation Consortium). 
The c.346G>C (p.G116R) variant identified in individual SATB2-01 is predicted to alter the wild type splice donor site for intron 4 (HSF 3.0) (9). Based on the American College of Medical Genetics and Genomics (ACMG) variant interpretation guidelines, 9 variants were classified as pathogenic (most or all of PVS1, PS2, PM2, PP3, PP4) (10). The remaining 2 missense variants identified in 3 individuals (p.R429Q and p.P655L) were classified as likely pathogenic using the same criteria (PS2, PM2, PP2, PP3, PP4).

Overall, the now 21 total variants reported in SATB2 are spread throughout the gene with only 3 instances of recurrent locations (Fig. 2A) (11). Selected phenotypic features that were less ubiquitous, such as the presence of cleft palate, absence of speech, or tibial bowing, were present in individuals regardless of the location of the pathogenic variants across the SATB2 protein domains and with variability even among those with the same variant. When looking at these 3 variable phenotypic features by the underlying type of pathogenic variant (nonsense, frameshift, missense, or splice site), cleft palate was more prevalent in individuals with frameshift (63\%) or nonsense (67\%) pathogenic variants compared to missense (17\%) alterations but this difference was not statistically significant $(\mathrm{p}=0.0544)$. For comparison, in 17 individuals with large deletions that include $S A T B 2$, tibial bowing has not been reported (but seen in $1 / 3$ individuals with intragenic duplications) while cleft palate has been described in 47\% (8/17) (1).

\section{Discussion}

SAS continues to be underdiagnosed condition. Six of the individuals with SATB2 pathogenic variants included in this cohort were diagnosed through WES performed at a single clinical laboratory that as of June 2016, has identified a total of 17 cases (including 4 previously reported) in a total of 6996 cases with ID/DD (0.24\%) (2). With this report, we have doubled the number of individuals described with SAS in the literature caused by point alterations. We provide further 
documentation of the main clinical features of this recognizable syndrome: universal developmental delay with severe speech difficulty, subtle facial dysmorphism, and a high frequency of craniofacial anomalies (micrognathia, palatal abnormalities, dental issues), behavioral issues, and brain neuroradiographic changes. This is in agreement with the recently proposed acronym of major features to help recognize this syndrome $(S$, Severe speech anomalies; $A$, Abnormalities of the palate; $T, T$ eeth anomalies; $B, B$ ehavioral issues with or without Bone or Brain MRI anomalies, and age of onset before 2 years of age; S.A.T.B.2) (1). While this phenotype appears to be more consistent and recognizable as individuals with SAS age, the high frequency of significant feeding difficulties (regardless of the presence of a cleft palate) and hypotonia suggest that this diagnosis should also be part of the differential diagnosis of infants with such problems, particularly if white matter abnormalities or delayed myelination are also documented on brain imaging.

Given the documented role of SATB2 in osteoblast differentiation, skeletal anomalies are to be expected in individuals with SAS (12). Of interest, tibial bowing, such as that seen in the individual here described, has now been reported in 3 individuals, all with truncating pathogenic variants $(3 / 24=12 \%)(5,13)$. The possibility that this skeletal phenotype is an age-dependent finding that results from dominant negative function, as recently postulated, remains unknown (5). Similarly, a high frequency of osteopenia in SAS is suggested by the several individuals with point pathogenic variants with this bone abnormality documented. Knowing the high frequency of osteopenia in SAS, two individuals underwent bone densitometry screening in this cohort with normal results. 
In agreement with the recent literature review of SAS, no individuals were documented to have genitourinary anomalies, major cardiac defects, or ectodermal changes (other than dental), findings that appear to be particularly prevalent in individuals with large deletions only (1).

The SATB2 gene encodes a protein of 733 amino acids with two CUT domains and a homeodomain, all highly conserved across vertebrate taxa (14). SATB2 functions as a transcription factor and a high level regulator of several gene regulatory networks with critical roles in multiple developmental processes $(12,15)$. Regardless of the location, haploinsufficiency and dominant negative effects have been suggested as potential pathomechanisms of point pathogenic variants in SATB2. In this study, we have identified additional missense variants to be causative of SAS, and their mechanism remains unknown. Of interest, four of the five reported missense variants are located within the first CUT domain of SATB2, a critical domain for the DNA recognition and binding required for SATB2 to regulate gene expression (Fig. 2B) (16, 17). Early genotype-phenotype correlations at this point suggest a lower frequency of cleft palate in individuals with missense variants. Regardless, for genetic counseling purposes, we anticipate near complete penetrance for the neurodevelopmental and dental alterations, and while germline mosaicism continues to be a theoretical possibility, no instances of inherited pathogenic variants have been documented thus far.

The present study does have some limitations. The retrospective nature of the study necessitates reliance on accurate and complete medical records, which may not be the case. Similarly, the use of an online survey with data entered by individuals or caregivers depends on their recollection of information. Lastly a larger sample is desirable to confirm some of the observations here discussed. 
In conclusion, SAS is a multisystemic disorder mainly characterized by significant neurodevelopmental compromise, behavioral issues, and craniofacial anomalies. As the recently proposed surveillance guidelines for individuals with SAS are adopted by providers, along with the circulation of greater resources about SAS (http://www.SATB2gene.com), further delineation of the frequency and impact of other phenotypic traits will become available. Similarly, as new cases of SAS are identified through WES or NGS technologies, further exploration of genotypephenotype correlations will be possible. 


\section{References}

1. Zarate YA, Fish JL. SATB2-associated syndrome: Mechanisms, phenotype, and practical recommendations. Am J Med Genet A 2017: 173: 327-337.

2. Zarate YA, Perry H, Ben-Omran T et al. Further supporting evidence for the SATB2associated syndrome found through whole exome sequencing. Am J Med Genet A 2015: 167A: 1026-1032.

3. Docker D, Schubach M, Menzel M et al. Further delineation of the SATB2 phenotype. Eur J Hum Genet 2014: 22: 1034-1039.

4. Leoyklang P, Suphapeetiporn K, Srichomthong C et al. Disorders with similar clinical phenotypes reveal underlying genetic interaction: SATB2 acts as an activator of the UPF3B gene. Hum Genet 2013: 132: 1383-1393.

5. Boone PM, Chan YM, Hunter JV et al. Increased bone turnover, osteoporosis, progressive tibial bowing, fractures, and scoliosis in a patient with a final-exon SATB2 frameshift mutation. Am J Med Genet A 2016: 170: 3028-3032.

6. Lee JS, Yoo Y, Lim BC et al. SATB2-associated syndrome presenting with Rett-like phenotypes. Clin Genet 2016: 89: 728-732.

7. Rauch A, Wieczorek D, Graf E et al. Range of genetic mutations associated with severe non-syndromic sporadic intellectual disability: an exome sequencing study. Lancet 2012: 380: 1674-1682.

8. Trakadis YJ, Buote C, Therriault JF et al. PhenoVar: a phenotype-driven approach in clinical genomics for the diagnosis of polymalformative syndromes. BMC Med Genomics 2014: 7: 22 . 
9. Desmet FO, Hamroun D, Lalande M et al. Human Splicing Finder: an online bioinformatics tool to predict splicing signals. Nucleic Acids Res 2009: 37: e67.

10. Richards S, Aziz N, Bale S et al. Standards and guidelines for the interpretation of sequence variants: a joint consensus recommendation of the American College of Medical Genetics and Genomics and the Association for Molecular Pathology. Genet Med 2015: 17: 405424.

11. Liu W, Xie Y, Ma J et al. IBS: an illustrator for the presentation and visualization of biological sequences. Bioinformatics 2015: 31: 3359-3361.

12. Dobreva G, Chahrour M, Dautzenberg M et al. SATB2 is a multifunctional determinant of craniofacial patterning and osteoblast differentiation. Cell 2006: 125: 971-986.

13. Leoyklang P, Suphapeetiporn K, Siriwan P et al. Heterozygous nonsense mutation SATB2 associated with cleft palate, osteoporosis, and cognitive defects. Hum Mutat 2007: 28: 732-738.

14. FitzPatrick DR, Carr IM, McLaren L et al. Identification of SATB2 as the cleft palate gene on 2q32-q33. Hum Mol Genet 2003: 12: 2491-2501.

15. Britanova O, Depew MJ, Schwark M et al. Satb2 haploinsufficiency phenocopies 2q32q33 deletions, whereas loss suggests a fundamental role in the coordination of jaw development. Am J Hum Genet 2006: 79: 668-678.

16. Niknafs N, Kim D, Kim R et al. MuPIT interactive: webserver for mapping variant positions to annotated, interactive 3D structures. Hum Genet 2013: 132: 1235-1243.

17. Yamasaki K, Akiba T, Yamasaki T et al. Structural basis for recognition of the matrix attachment region of DNA by transcription factor SATB1. Nucleic Acids Res 2007: 35: 50735084. 


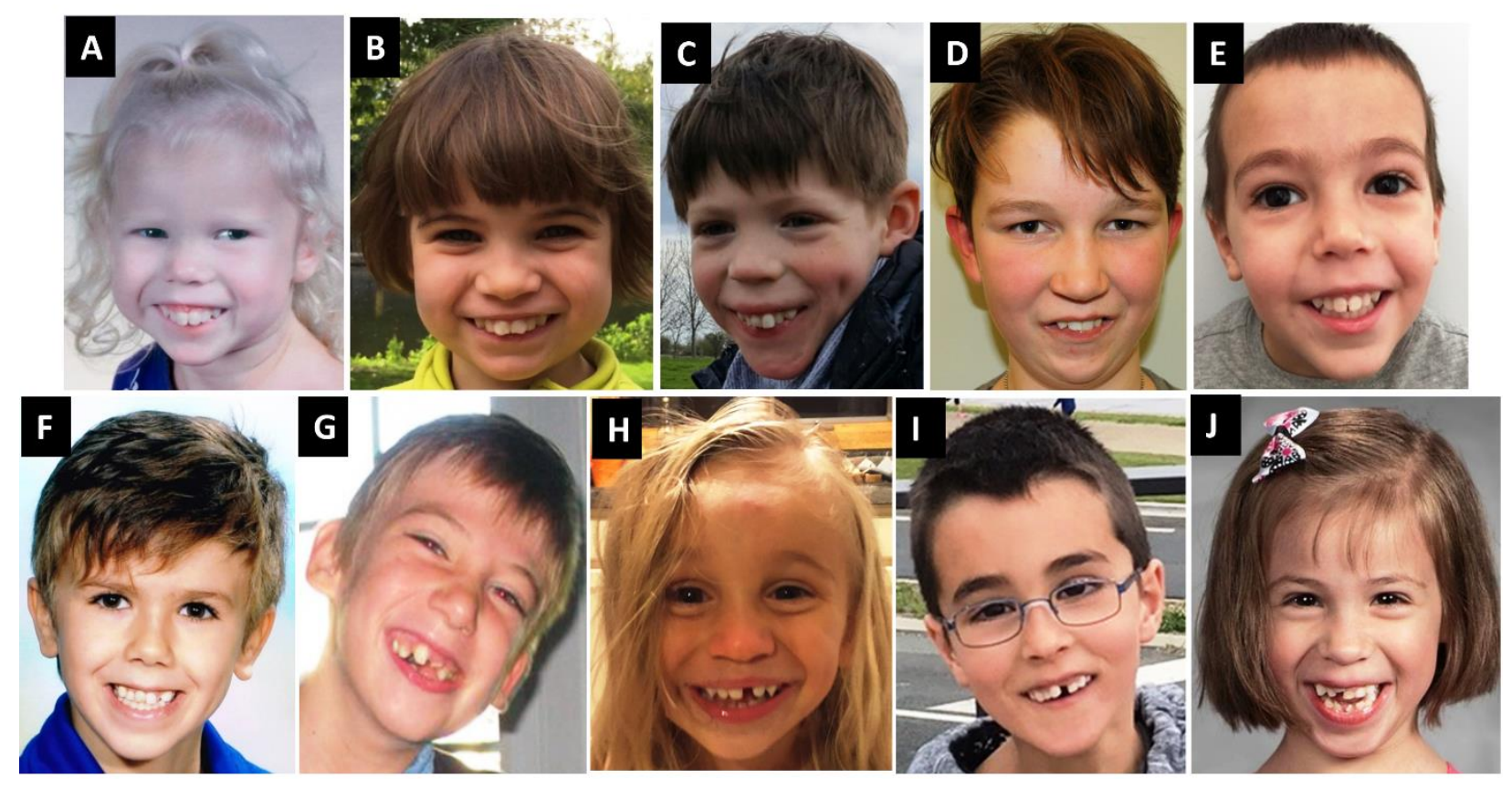

Fig. 1. Frontal facial photographs of ten individuals with SAS included in the study. A: Individual SATB2-01, B: Individual SATB2-05, C: Individual SATB2-16, D: Individual SATB2-10, E: Individual SATB2-04, F: Individual SATB2-06, G: Individual SATB2-14, H: Individual SATB2-23, I: Individual SATB2-07, J: Individual SATB2-18. Note the clinical similarities including long and/or flat philtrum, deeply set eyes, thin vermillion of the upper lip, and prominent upper frontal incisors. 
A

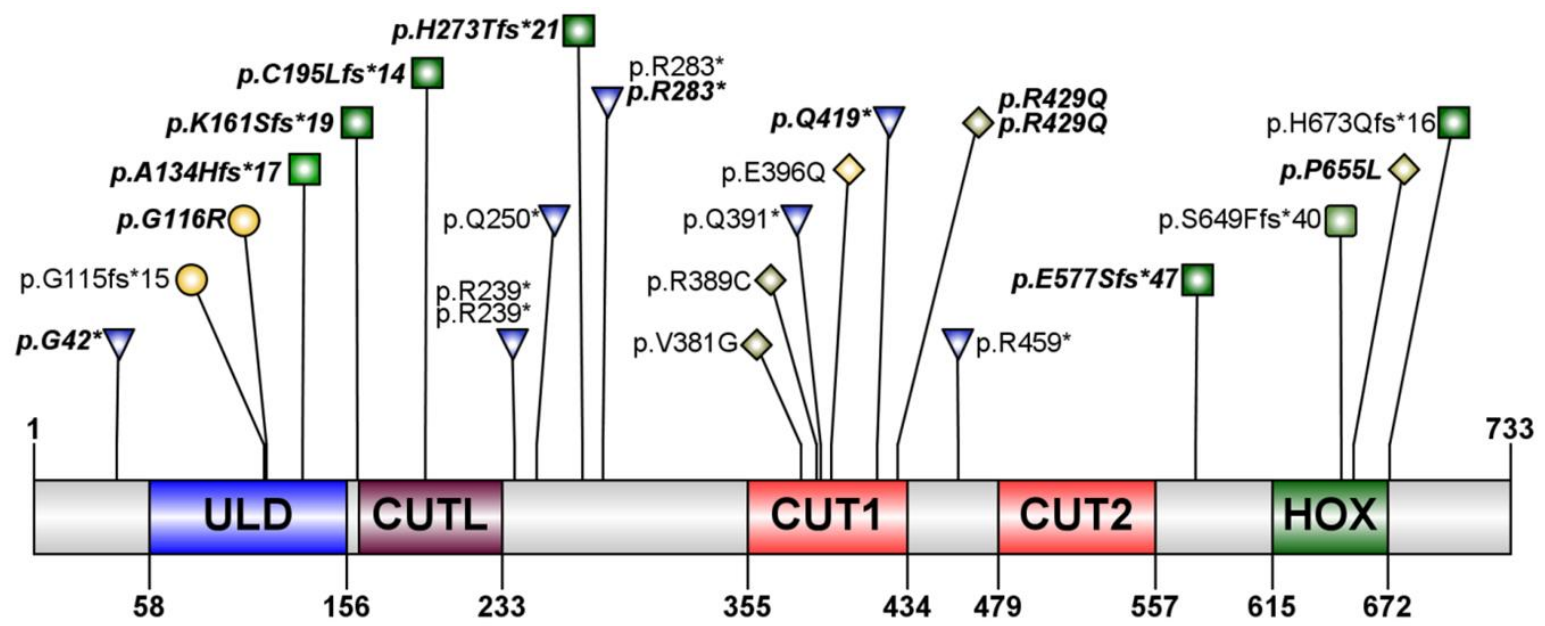

B

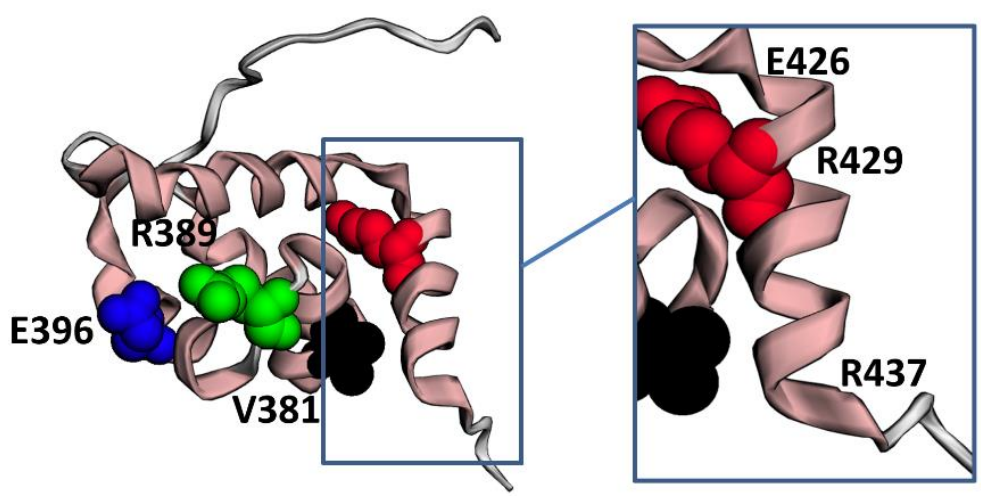

Fig. 2. A. Schematic representation of SATB2 and its functional domains according to Pfam.

Arabic numerals correspond to amino acid number. Diagrams were constructed using Illustrator for Biosequence (IBS1.0.1). Variants listed as black font were previously reported. Variants listed as italic bold font are from this report. Variants are represented with triangles (nonsense), circles (splice site), squares (frameshift) or diamonds (missense). Abbreviations are as follows: ULD, ubiquitin-like oligomerisation, CUTL, CUT1-like DNA binding; HOX: homeobox. B. Structural representation of the CUT1 DNA binding domain with the residues affected by missense variants. Previously reported variants include p.V381G (black), p.R389C (green), and p.E396Q (blue). The p.R429Q variant identified in two individuals in this cohort (red) is located in the $\alpha 5$ helix. Starting and ending residues of the $\alpha 5$ helix are also illustrated. 